\title{
THE EFFECT OF CORRECTIVE EXERCISES ON THE RAMGE OF MOTION OF THE HIP JOINTS AND THE RESULT OBTANIED IN THE DEEP SQUAT OF FMS TEST
}

\author{
Jolanta Oleksiak, 1, 3, A, B, D, E Anna Sobianek, 2, 3, A, E Maciej Janiszewski' 3, A, B, D \\ ${ }^{1}$ Department of Heart Failure and Cardiac Rehabilitation of the Warsaw Medical University \\ ${ }^{2}$ Department of Physical Education and Sport of the Warsaw Medical University \\ ${ }^{3}$ II Faculty of Medicine with the Division of Teaching in English and the Department of Physiotherapy of the Warsaw Medical University \\ A Study Design; ${ }^{\mathrm{B}}$ Data Collection; ${ }^{\mathrm{D}}$ Manuscript Preparation; E Funds Collection
}

\author{
Address for corpespondence: \\ Oleksiak Jolanta \\ Witosa 4/3, 07-200 Wyszków, Poland \\ E-mail: oleksiak.jolanta@gmail.com
}

\begin{abstract}
Ahstract Squat is a fundamental motor pattern in everyday activities, e.g. sitting or lifting objects from the ground. One of the reasons for the poor quality of the squat pattern lies in limited mobility. The study assessed the effects of one-off and 4-week squat correction based on the mobility system exercises. The study involved 40 people, divided into a test group (18 women, 2 men, \pm 24.6 years) and a control group ( 10 women, 10 men \pm 23.6 years). All participants have been subjected to three tests twice: a deep squat test - according to the FMS ${ }^{\mathrm{TM}}$ concept, the Active Straight Leg Raise test and the "four" test. After the test, four corrective exercises were recommended and the study continued. The subjects from the test group performed the recommended exercises for 4 weeks, 3 times a week. The protocol was repeated for both groups after 4 weeks, according to the same methodology. For the ASLR test and the "four" test, a significant improvement was observed both after a single session and after a 4-week correction program. With the improvement of these ranges, the result in the deep squat test also improved. A statistically significant improvement in the result was obtained in 9 out of 40 people $(22.5 \%)$ after a single measurement. As a result of a 4-week correction, the improvement of the squat occurred only after repeated correction. The use of corrective exercises in the hip joints may translate into an improvement in the squat pattern.
\end{abstract}

Key Wordls squat, mobility, range of motion, foam rolling, stretching

\section{Introduction}

Sitting is one of the basic movement patterns used in everyday life. However, this seemingly natural activity, performed incorrectly, dozens of times a day, can become the cause of overload changes and lead to permanent degenerative changes in the musculoskeletal system (Mankin, Treadwell, 1986). Biomechanics of sitting is strongly related to the mobility of the hip joint. This joint generates the greatest forces, and the work and tension of the muscles included in the entire osteo-joint-muscular syndrome affects the quality of the squat pattern. FMS ${ }^{\mathrm{TM}}$ Test 
is one of the most popular tests used to assess the squat pattern. Currently, mobility exercise system that uses and combines stretching, auto-massage and joint mobilization techniques is a widely known tool used to increase mobility.

\section{The Concept of Mobility}

Mobilization, defined as movement-based, integrated approach to the human body, taking into account all the elements potentially limiting its motor skills, is a relatively modern concept existing in the space of strengthendurance training and physiotherapy. It can be said that mobilization is a tool that allows to move and practice more effectively. From this point of view, it is a system of conduct that can be dedicated both to people who deal with sport professionally, but also to people who train recreationally, and even to people who do not deal with physical activity, e.g. as an element of rehabilitation programs. To understand the essence of novelty represented by the mobility exercise system, a distinction should be made between the concept of flexibility and the concept of mobility. Flexibility is the ability of a man to achieve a large amplitude in movements. Exercise of flexibility is to prepare the movement apparatus for the implementation of the starting tasks and to master the rational technique (Hara et al., 2016).

It turns out that in sports, training flexibility alone is not enough. The own experience of many coaches and the players themselves shows that training flexibility itself, in all its generality and breadth, does not necessarily translate into benefits in the form of better results in a given discipline (Marciniak, 1998). Although being important, the passive range of motion is not enough to achieve high results in spor.

In addition to strength in the final range of movement, mobility is made up of one more value - an aspect emphasized by Moshe Feldenkrais, as an inherent feature of all fully aware movement activities. It is about reversibility, i.e. the possibility of volitional stopping a given movement at any moment and withdrawing or modifying it (Rurak, 2013). The central nervous system (CNS) efficiency plays a key role in this mechanism.

In summary, the components of mobility can be defined as follows:

$$
\text { mobility }=\text { passive mobility }+ \text { strength in the extreme range of motion + motor control }
$$

A full understanding of the definition of "mobility" is possible only after a detailed analysis of the motoric feature, which is flexibility and methods of its formation.

Mobility should be understood as a wider notion- not as a single motor feature, but rather a collection of several such features. According to the theory of mobility, there is no one, universal way to correct the limits of movement ranges, loosening tense muscles and treating sore joints. To deal with any problems related to soft tissue stiffness, it is necessary to apply methodological approach and combine several different techniques. The idea of this system is to use as many mobilization techniques as possible, until the limited positions are corrected and the pain is eliminated.

\section{Material and methods}

The study included a group of 40 people, aged 20-30, 29 women and 11 men. The subjects were divided into two non-statistically different groups: test $(\mathrm{A})$ and control $(\mathrm{B})$.

Exclusion criteria included: the presence of pain in the hip joints, past injuries, injuries or surgical procedures that could affect the movement of the lower limbs. 
The division of subjects into the test or control group was carried out randomly. After recruitment for the study, a total of three functional tests were carried out on all subjects:

1. The test of deep squat - according to the FMS ${ }^{\mathrm{TM}}$ concept.

2. Active Straight Leg Raise test.

3. The "four" test.

The deep squat test was assessed according to the standards of the FMS concept. This test was created and named (Functional Movement System) by the American physiotherapist Gray Cook (Aptowicz, 2010). In the ASLR test (Active Straight Leg Raise) the ability to actively lift a straight leg with a stabilized trunk was evaluated. The results were recorded by measuring the goniometric flexion in the hip joint. The last test, which was performed, was the "four" test, measuring the range of external rotation of the hip joint, name after the position of the lower limbs of the examined person, which resembles the number 4 . After performing a functional examination using the above-described tests, the subjects were instructed to do four correction exercises.

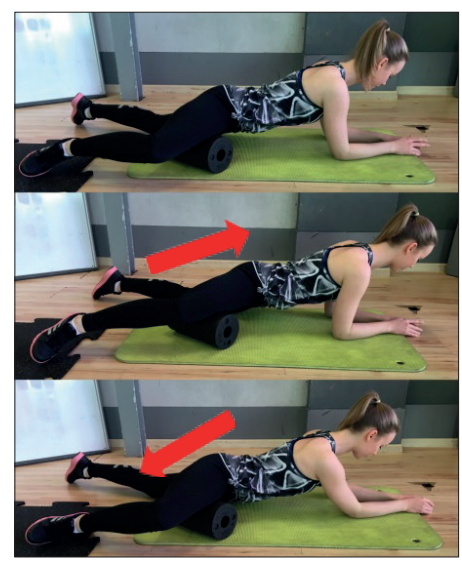

a)

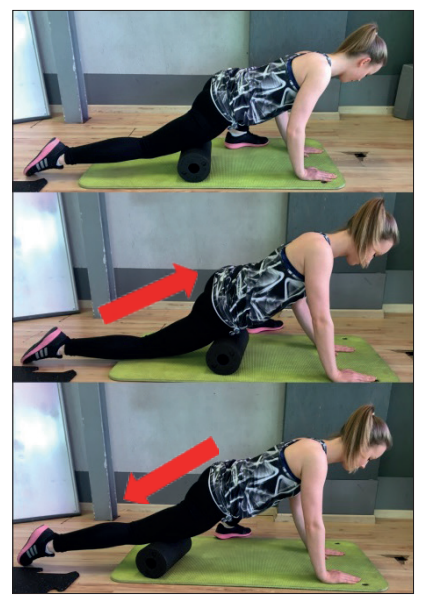

c)

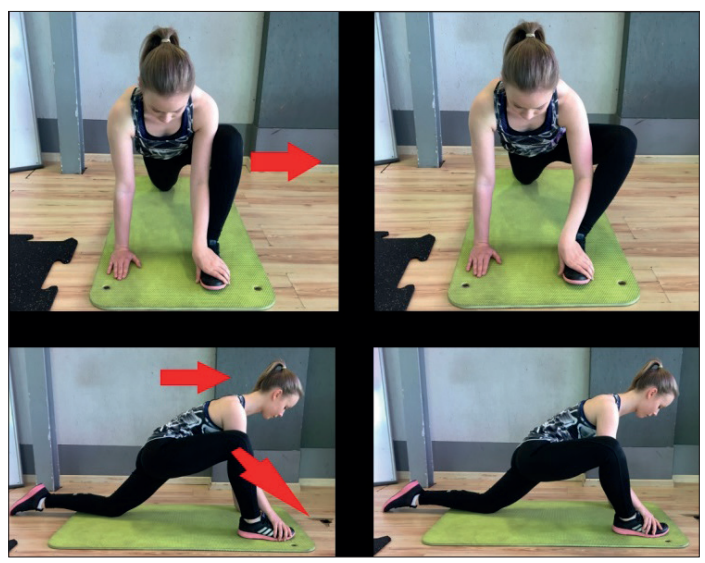

b)

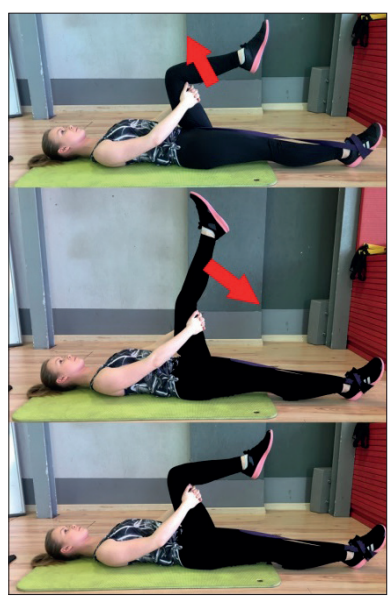

d)

Figure 1. Corrective exercises used in the course of the study 
Exercise 1 - Breaking the muscles of the adductor with a roller (Figure 1a), involving the loosening of the muscle group with a foam roller.

Exercise 2 - Bending of one leg with external rotation (Figure 1b) - exercise using dynamic muscle stretching from the group of adductors, ilio-lumbar muscles, deepening the external rotation of the hip joint in a position close to the position of the lower squat.

Exercise 3 - Breaking quadriceps muscles with roller (Figure 1c), which involves loosening the muscle group with a foam roller.

Exercise 4 - Classic mobilization with anatomical back tape (Figure 1d), using dynamic muscle stretching from the ischiocrural group.

The purpose of the above exercises was the normalization of muscle tone of the muscles of the ischiocrural group, the muscles of the adductors and the quadriceps muscle of the thigh.

The main aim of the research was to examinate the influence of this exercises on hip range of motion and compare it with the deep squat FMS ${ }^{\mathrm{TM}}$ test.

Both people in group $A$ and people in group $B$, after performing corrective exercises, were again subjected to functional tests. Test group (A) performed above 4 exercises for 4 weeks, 3 times a week, while control group (B) did not perform any corrective exercises during these 4 weeks. After 4 weeks from the first examination and correction, the subjects in both groups were tested again, using the same measurement methodology and taking into account the same corrective exercises.

Methodology of conducted tests

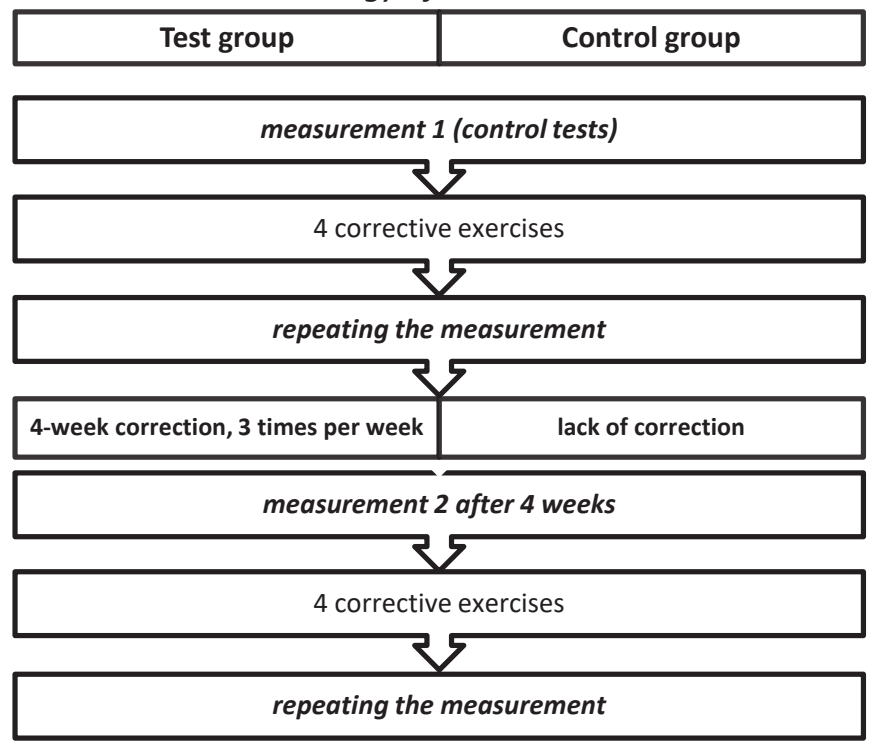

Figure 2. Methodology of conducted tests 
The study of empirical data involved standard statistical analysis methods. Kolmogorov-Smirnov test was used to calculate the distribution of normality. The distribution was not normal in all groups. The results of individual tests were presented using arithmetic averages, taking into account standard deviations. Significance of differences between the pairs of variables was evaluated by Student's t-test and Levene's test for normal distributions and U Mann-Whitney test for abnormal distributions, for a minimum significance level of $p<0.05$.

\section{Results}

During the first measurement there was a statistically significant improvement in the range of motion after correction in all subjects, both in the external rotation measured by the "four" test and active hip flexion in the ASLR test for both lower limbs and the result in the deep squat test FMS ${ }^{\text {TM }}$ (Table1).

Table 1. Differences $(p)$ between the results of individual tests, performed after a single correction

\begin{tabular}{lc}
\hline \multicolumn{1}{c}{ Tests } & Significance $(\mathrm{p})$ \\
\hline FMS ${ }^{\mathrm{TM}}$ squat & 0.003 \\
SLR L Test & 0.000 \\
SLR P Test & 0.000 \\
„Four” Test L & 0.012 \\
„Four” Test P & 0.016 \\
\hline
\end{tabular}

In the case of the squat test, the score was improved in 9 out of 40 people (22.5\%). In 31 (77.5\%) people there was no change in the result, while deterioration was observed in no case. The arithmetic mean of this test before the measurement was 2.300 , and after measurement 2.5250. The average deviation from the mean was for the test before correction 0.60764 , and for the test after correction 0.55412 (Figure 3). The statistical significance was obtained in the analysis at the level of $p=0.003$ (Table 1).

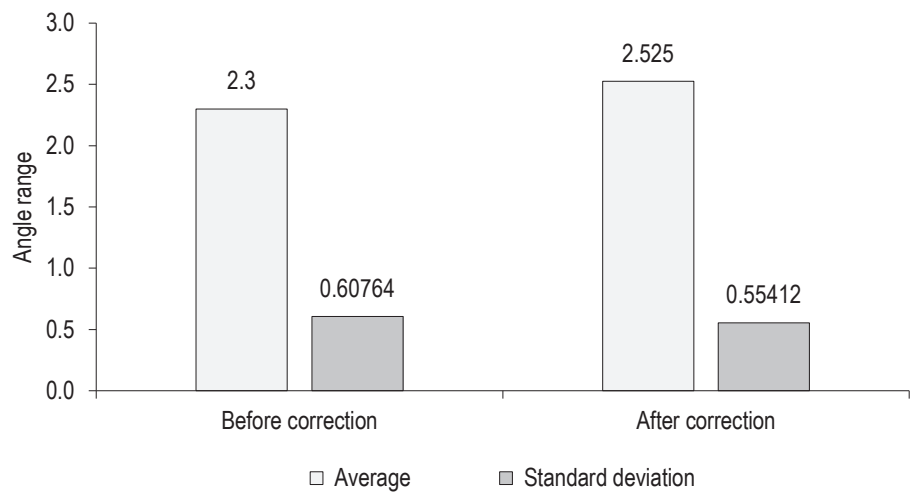

Figure 3. Difference in mean results obtained in the deep squat FMS test after a single correction 
The greatest improvement was recorded for the ASLR test in the right limb. For 40 people, 33 obtained a better result in the post-correction test (82.5\%). In 5 people (12.5\%) no change occurred, and in 2 people (5\%) the result was worse. The arithmetic mean of the obtained results before correction was 75.8750 and after correction 80.5250. The standard deviation for the above tests was 9.13485 and 9.12024 respectively (Figure 4).

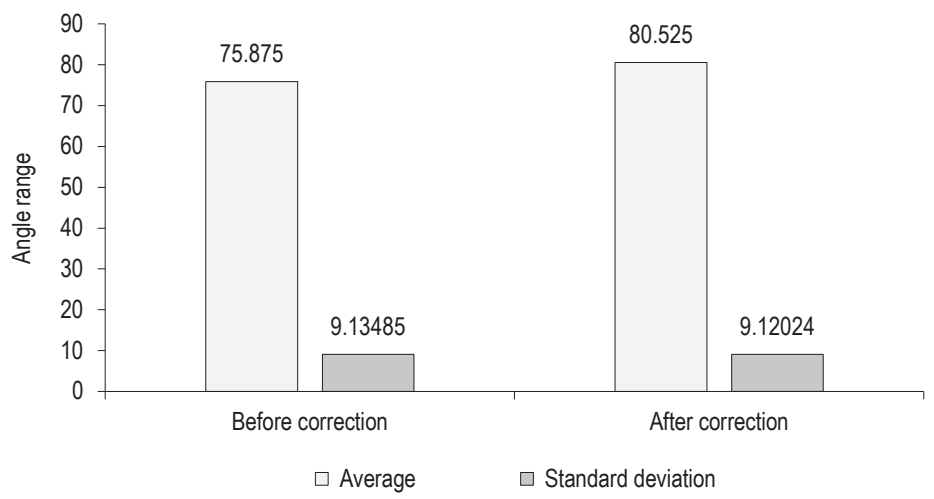

Figure 4. Difference in mean results obtained in the SLR test for the right lower limb after a single correction

The second analysis performed was a comparison of the results from the first measurement before the correction and the second measurement (after 4 weeks) both before and after the correction (Table 2). The results of the second measurement before the correction were analyzed in order to exclude the physiotherapist's influence on the effect of 4 -week correction in the test group.

Table 2. Differences [p] between the results of individual tests obtained in measurement 1 and measurement 2 before and after correction

\begin{tabular}{|c|c|c|}
\hline \multirow{2}{*}{ Tests } & \multicolumn{2}{|c|}{ Significance (p) } \\
\hline & before correction & after correction \\
\hline $\begin{array}{l}\text { Pair } 1 \\
\text { SLR L Test (1) - SLR L Test (2) }\end{array}$ & 0.007 & 0.000 \\
\hline $\begin{array}{l}\text { Pair } 2 \\
\text { SLR P Test (1) - SLR P Test (2) }\end{array}$ & 0.005 & 0.000 \\
\hline $\begin{array}{l}\text { Pair } 3 \\
\text { "Four" Test L (1) - "Four" Test L (2) }\end{array}$ & 0.012 & 0.003 \\
\hline $\begin{array}{l}\text { Pair } 4 \\
\text { "Four" Test P (1) - "Four" Test P (2) }\end{array}$ & 0.022 & 0.073 \\
\hline $\begin{array}{l}\text { Pair } 5 \\
\text { FMS squat (1) - FMS squat (2) }\end{array}$ & 0.480 & 0.007 \\
\hline
\end{tabular}


Statistical analyzes showed a significant difference between the results obtained during the first measurement before correction and the second measurement before the correction for both limbs in ASLR tests and in the "four" test. However, no significant changes were found for the squat test (Table 2).

Finally, the results obtained by the test and control group were analyzed by juxtaposing them together. In the course of the conducted analyzes, there was no significant difference between the test group and the control group in the range both before and after correction for any of the tests.

\section{Discussion}

Squat is an ideal test, checking the presence of functional disorders in the human body. Based on the observations and video analysis of the squat, one can find weak points in the bio-kinematic chain, and then make the correction of the pattern disorders. Learning squat technique is a long-term process. It often requires the use of additional exercises, activating weak muscle groups (gluteus maximus, medius, ischiocrural group, abdominals, stabilizers of the shoulder), mobility exercises (ankle, hip joint, spine, shoulder) and stretching (gastrocnemius and soleus muscle, ischiocrural group, hip flexors, hip rotators, backbone rectifier muscle, quadratus lumboeum, latissimus dorsi, pectoralis major and minor) (trenerindywidualny.pl, 2014). The main purpose of this work was to examine whether the range of mobility of the hip joints influences the result obtained in FMS ${ }^{\mathrm{TM}}$. The obtained results showed that along with the improvement of these ranges, the result in the deep squat test was also improved, however it was more expressed for the ASLR test than for the "four" test. However, no statistical confirmation of this conclusion was obtained.

Studies have shown that properly selected exercises, aimed at eliminating the cause of limitation after a single correction, give the statistically significant improvement in ranges of mobility for both hip flexion (an active straight leg lift) and external rotation ("four" test). There was also a significant improvement in the results obtained in the deep sit-up test according to FMS $^{\mathrm{TM}}$, which may mean that the above-mentioned ranges of motion have a significant impact on the quality of this pattern.

The squat test obtained after 4 weeks of exercising shows an interesting result. In the test before re-correction, no statistical significance was achieved in terms of improvement in relation to the first measurement. This was achieved only after a new correction, which may mean that the improvement of hip mobility, obtained through 4 weeks of exercise, was insufficient to improve the mobility pattern. Only performing the test immediately after correction resulted in a significant improvement.

The above observation suggests that the effects of the applied correction are short-term changes, so performing them "as a spare" will not translate into a general improvement in the quality of the squat.

Similar conclusions were drawn by the authors of other publications regarding the effect of myofascial relaxation and stretching on the range of motion (Davis, 2005; Kiestler, 2010; O'Sullivan, 2009). The study of 2014, Jay et al. showed a significant improvement in active flexion in the hip joint after using a 10-minute session of muscle massage of the ischiocrural group using a roller. However, it was observed that this effect lasted only 10 minutes after the end of the session.

In a study conducted by Macdonald et al. in 2014, the effectiveness of foam-rolling was demonstrated in a group of 20 active men who were ordered to perform a protocol consisting of 10 sets of 10 repetitions of back squats. The results showed a significant increase in the mobility of the hip (72 h after) and knee joint (48 and $72 \mathrm{~h}$ after). 
Moteiro et al., in their work on the deep squat test according to FMS ${ }^{\mathrm{TM}}$, analyzed how the length of selfmassage technique of selected areas of the body affects the result in the FMS ${ }^{\mathrm{TM}}$ test. For one group the test was preceded by self-massage of the lateral side of the thigh (iliotibial band) with a foam roller, and for the second group by self-massage of the lateral torso (latissimus dorsi) using a foam-roller and plantar side of the foot (plantar aponeurosis) using tennis ball. In his results he obtained a statistically significant improvement of the squat for both groups and noted that the minimum time for obtaining significant changes in the time of performing the automassage is 90 seconds.

Sullivan et al. also analyzed the effect of rolling on the length of the rear thigh muscle group, measured by the sit and reach test. The examined group of people was subjected to massage with a special rolling device for 5 and 10 seconds respectively. The results showed a $4.3 \%$ increase in the range of mobility in the sit and reach test for the group which was massaged longer and $2.3 \%$ for the group with shorter massage. In addition, the group underwent an EMG examination, which did not show significant changes in the nervous excitability of the muscle group being tested.

A similar study was conducted in 2015 by Bradbury-Squires et al., where a group of recreationally practicing, middle aged men underwent quadriceps massage using a roll massager for one group for $5 \times 20$ seconds, and for the other for $5 \times 60$ seconds. In the results of these studies, $10 \%$ improvement for the first group and for the second $16 \%$ improvement in bending range in the knee joint was observed.

The above studies have been quoted to show the effect of the time unit on the effects of myofascial relaxation. They are extremely important and may also confirm the assumptions of this work, which indicate that a significant part of the disorders related to the squat pattern is associated with soft tissues, and therefore myofascial work will be very helpful in the processes of its reeducation.

However, there are studies that contradict the above-mentioned reports. Couture et al. did not show a significant relationship between the time of self-massage and the range of mobility in the knee joint. Moreover, no differences were noted in the ranges as compared to the control group that did not perform the test protocol. It is worth noting that the differences occurring in reports on the effectiveness of foam-rolling can be the result of differences in the tools used (different hardness rollers), intensity (value) of pressure during the execution of individual techniques and methods of measuring joint mobility.

In addition to foam-rolling, the mobility system also uses techniques for stretching and mobilizing the joint using a tape.

Exercises used in this study have been selected in such a way as to increase the elasticity of the ischiocrural group by means of dynamic (active) stretching. A significant improvement in the scope of active straight leg raise (ASLR) was obtained both after a single session and after a 4-week correction program. However, no significant difference was found between the two measurements. The group that performed exercises regularly obtained a partial fixation of the improved ranges, while they were not significantly greater than those achieved in a single session, which may mean that the results obtained during the exercise have a limited range of effectiveness.

Avala et al. shared similar conclusions in studies, which investigated the effect of active stretching of the ischiocrural muscles on the passive flexion range in the hip. The results showed a $26.0 \pm 8.8 \%$ improvement in the range of passive flexion in the hip joint after the end of the protocol. However, it has not been demonstrated that there is a significant difference between measurements during the entire protocol. On the other hand, significant regress was observed both 2 and 4 weeks after the end of the protocol. 
The results of the present research may be criticized due to the lack of precise research methods, based largely on the subjective assessment of the researcher (FMS ${ }^{\text {TM }}$ Test) and with a high risk of measurement error in the context of registration of motion ranges. It should be noted that they have been selected in a way to be easy to perform in non-laboratory conditions. Nevertheless, the obtained results are a reason to conduct more detailed studies in which a larger group of people would be tested, and the measurement methods would be more sensitive.

In addition, it seems important to conduct research in which the two above-described methods: foam-rolling and stretching combined with the mobilization of the joint with tape, would be separated. In the present study, it cannot be unequivocally determined which of the applied methods turned out to be more effective.

\section{Conclusions}

1. The mobility system, which consists of: self-massage, dynamic stretchning and joint mobilization with the help of tape, are an effective method of increasing the ranges of mobility of the hip joints.

2. Greater mobility of the hip joints results in a higher score from the deep squat FMS ${ }^{\text {TM }}$ test.

3. Even a single correction allows to improve ranges of movement and the squat pattern.

4. Regularly performed corrective exercises do not translate into a better result than in one-off correction.

\section{References}

Aptowicz, J. (2010). Zastosowanie FMS. (Functional Movement Screen) do oceny ruchomości funkcjonalnej na przykładzie zawodników uprawiających taekwondo olimpijskie. Olsztyn: Olsztyńska Szkoła Wyższa im. Józefa Rusieckiego, Tom 10 (4).

Avala, F., Sainz, De Baranda, P., De Ste Croix, M. (2010). Effect of active stretch on hip flexion range of motion in female professional futsal players. J Sports Med Phys Fitness, 50 (4), 428-435.

Bradbury-Squires, D.J., Noftall, J.C., Sullivan, K.M., Behm, D.G., Power, K.E., Button, D.C. (2015). Roller-massager application to the quadriceps and knee-joint range of motion and neuromuscular efficiency during a lunge. J Athl Train, 50 (2), 133-140.

Couture, G. (2015). The Effect of Foam Rolling Duration on Hamstring Range of Motion. The Open Orthopaedics Journal, 9, 450-455.

Davis, D.S., Ashby, P.E., McCale, K.L., McQuain, J.A., Wine, J.M. (2005). The effectiveness of 3 stretching techniques on hamstring flexibility using consistent stretching parameters. J Strength Cond Res, 19 (1), 27-32.

Hara, D., Nakashima, Y., Hamai, S., Higaki, H., Ikebe, S., Shimoto, T., Yoshimoto, K., Iwamoto, Y. (2016). Dynamic hip kinematics in patients with hip osteoarthritis during weight-bearing activities. Clin Biomech (Bristol, Avon), 32, 150-156.

Jay, K., Sundstrup, E., Sondergaard, S.D., Behm, D., Brandt, M., Saervoll, C.A., Jakobsen, M.D., Andersen, L.L. (2014). Specific and cross over effects of massage for muscle soreness: randomized controlled trial. International Journal of Sports Physical Therapy, 9, 82-89.

Kistler, B.M., Walsh, M.S., Horn, T.S., Cox, R.H. (2010). The acute effects of static stretching on the sprint performance of collegiate men in the 60- and 100-m dash after a dynamic warm-up. J Strength Cond Res, 24 (9), 2280-2284.

Macdonald, G.Z., Button, D.C., Drinkwater, E.J., Behm, D.G. (2014). Foam Rolling as a Recovery Tool after an Intense Bout of Physical Activity. Medicine \& Science in Sports \& Exercise, 46 (1), 131-142.

Mankin, H.J., Treadwell, B.V. (1986). Osteoartritis: a 1987 update. Bull Rheum Dis, 36 (5), 1-10.

Marciniak, J. (1998). Zbiór ćwiczeń koordynacyjnych i gibkościowych. Warszawa: Centralny Ośrodek Sportu.

Monteiro, E.R., Škarabot, J., Vigotsky, A.D. (2017). Acute effects of different self-massage volumes on the FMS ${ }^{\text {TM }}$ overhead deep squat performance. Int J Sports Phys Ther, 12 (1), 94-104.

O'Sullivan, K., Murray, E., Sainsbury, D. (2009). The effect of warm-up, static stretching and dynamic stretching on hamstring flexibility in previously injured subjects. BMC Musculoskelet Disord, 10, 37.

Rurak, P. (2013). Gibkość, ruchomość i mobilność. Retrieved from: http://paulpipers.pl/blog/2013/11/gibkosc-ruchomosc-i-mobilnosc (15.03.2017). 
Sullivan, K.M., Silvey, D.B.J., Button, D.C., Behm, D.G. (2013). Roller-massager application to the hamstrings increases sit-and-reach range of motion within five to ten seconds without performance impairments. Int J Sports Phys Ther, 8 (3), 228-236.

Trener Indywidualny. Przysiad - ćwiczenie fundamentalne. Retrieved from: http://www.trenerindywidualny.pl/2014-05-05-12-58-36/ artykuly-trening-silowy/1636-przysiad-cwiczenie-fundamentalne.html (27.02.2017).

Cite this article aS: Oleksiak, J. Sobianek, A., Janiszewski, M. (2019). The Effect of Corrective Exercises on the Range of Motion of the Hip Joints and the Result Obtained in the Deep Squat of FMS Test. Central European Journal of Sport Sciences and Medicine, 2 (26), 31-40. DOI: 10.18276/cej.2019.2-03. 